\title{
Anatomical Study of Red-eared TurteTail (Trachemys scripta elegans)
}

\author{
Putri Nofita ${ }^{1}$, Muhammad Ja'far Luthfi ${ }^{2}$, Reza Sukma Dewi ${ }^{1 *}$ \\ ${ }^{1}$ Biology Department, ${ }^{2}$ Biology Education Department, Faculty of Science and Technology, UIN Sunan Kalijaga \\ Jl. Marsda Adisucipto No 1 Yogyakarta 55281, Indonesia. Tel. +62-274-540971, Fax. +62-274-519739 \\ "Email: ezasarear654@gmail.com
}

\begin{abstract}
Turtles are reptiles armored backs hard, slow motion, appearing for the first time about 200 million years ago and relatively unchanged for 150 million years. This study aims to determine the anatomical structure of the axial skeleton and determine the histologic structure of red-eared turtles (Trachemys scripta elegans) females. The method used, among others, Alizarin Red S staining Alcian Blue, methods of paraffin with Hematoxylin-eosin staining (HE) and Mallory acid fuchsin and used 3 adult turtles 3 years old. The results showed that vertebral caudalis red-eared turtles (Trachemys scripta elegans) females composed of true bone, vertebrae-type amphicoelous not have plains autotomy and histology red-eared turtles (Trachemys scripta elegans) females resemble histological structure other tail reptiles with some differences that only have 4 files do not have the muscle and fat tissue.
\end{abstract}

Keywords: Alizarin Red S Alcian Blue, Hematoxylin-eosin (HE), Mallory acid fuchsin, Red-eared turtle tails

\section{INTRODUCTION}

The turtles are reptiles armored backs hard, slow motion, appearing for the first time about 200 million years ago and relatively unchanged for 150 million years. These animals are very easy to identify from the shield that wraps the body similar to the box. Only the legs, head and tail are sticking out. In addition, these animals have no teeth and instead turtles have jaws closed edges sharp horn substance (Encyclopedia of Indonesia, 1988).

Abundant biodiversity of flora and fauna make the creation of a variety of species that have structural characteristics that distinguish the anatomy and morphology of the species with other species. It also makes reptiles, especially turtles have an anatomical structure and morphology that are different from other animals (Encyclopedia of Indonesia, 1988).

In terms of structure, reptiles supported by the tail vertebrae (vertebrae caudalis) are arranged in rows from the base to the tip of the tail. The tail is also equipped with perivertebral fat tissue (at the edge of the tail bone), muscle, nerves (central and edge), blood vessels and skin along with compactus scale (Susilo, 1999). The structure of the tail vertebrae have arranged segmental muscle layer surrounding fatty tissue, blood vessels located in the ventral part of the vertebrae and skin (Susilo, 1992).

Tail structure of the reptiles have a high variation related to the environment and behavior. The tails of most reptiles serves to avoid themselves from their enemies. There are several ways that are often used to avoid predators such as: camouflage, hide, escape and autotomy (Curtis, 1983). The purpose of this study are as follows knowing the anatomical structure of the axial skeleton red-eared turtles (Trachemys scripta elegans) females and histological structure.

\section{MATERIALS AND METHODS}

Making the vertebra intact preparet caudalis with staining method Alizarin Red S-Alcian Blue (Inouye, 1976). Making preparations for the longitudinal and transverse slices using Hematoxylin eosin staining (HE) (Suntoro,1983). Making preparations for the longitudinal and transverse slices using acid fuchsin staining method Mallory (Suntoro, 1983).

\section{RESULTS AND DISCUSSION}

Macroscopic Observation of Anatomy Red-eared Turtles (Trachemys scripta elegans) Females

In making preparations for a whole shows that the vertebra caudalis Red-eared turtles (Trachemys scripta elegans) females by staining Alizarin Red S-Alcian Blue is composed of true bone and cartilage do not have. Therefore red bone. This is because the process of forming the red color can occur because of a dye that is given is bound or absorbed by the calcium ions contained in the bone matrix. The more red color means the content of calcium ions in the bone more and more.

In Red-eared turtles (Trachemys scripta elegans) females by staining Alizarin Red S-Alcian Blue (Figure 1) is especially noticeable in parts of vertebrae seen chevron, neural arch and centrum as supporters of the tail down. This suggests that the bone perfectly smeared with red color indicates that the axial skeleton Red-eared turtles (Trachemys scripta elegans) female ossification process is already occurring in the presence of calcium in the bones. According to Kiernan (1981) red color from staining with Alizarin Red S-Alcian Blue dye produced from the bond Alizarin Red S-Alcian Blue with calcium ions. 


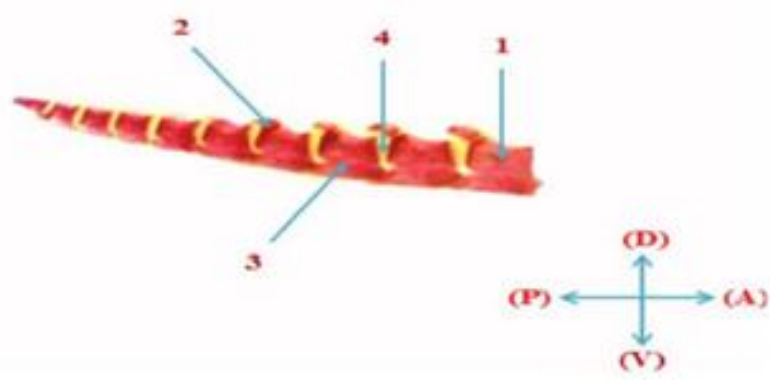

Figure 1. Mixture bone intact red-eared turtles (Trachemys scripta Elegans) females by staining Alizarin Red S-Alcian Blue (as seen from the anterior side or front view). Dorsal (D), Ventral (V), Anterior (A), Posterior (P). Centrum (1), Chevron (2), Neural arch (3), Intervertebral (4).

On the whole preparation red-eared turtles (Trachemys scripta elegans) females (Figure 2) red in the skeleton has been through, means all parts of the skeleton has undergone ossification. Mixture red-eared turtles (Trachemys scripta elegans) females by staining Alizarin Red S-Alcian Blue (Figure 2) also shows composed by bone tissue and seen prosessus lateralis, chevron bones and centrum.

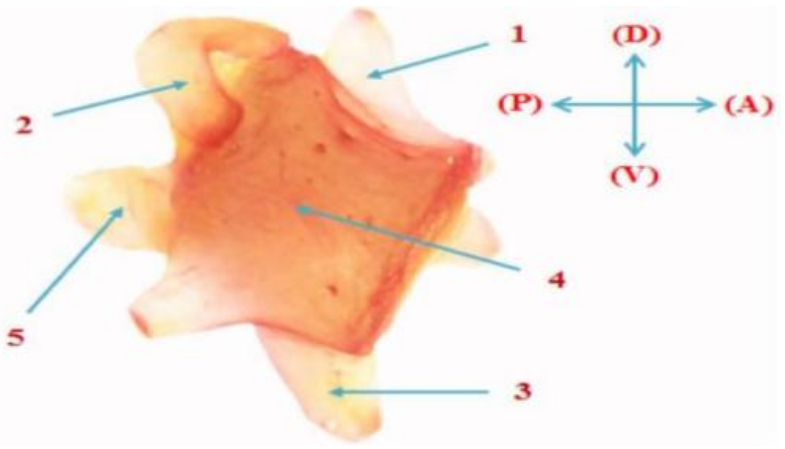

Figure 2. Mixture bone intact red-eared turtles (Trachemys scripta elegans) females by staining Alizarin Red S-Alcian Blue (as seen from the ventral side or looks down). Dorsal (D), Ventral (V), Anterior (A), posterior (P). Prosessus lateralis (1), Bone chevron (2), Prezygapophysis (3), Centrum (4), Postzygapophysis (5).

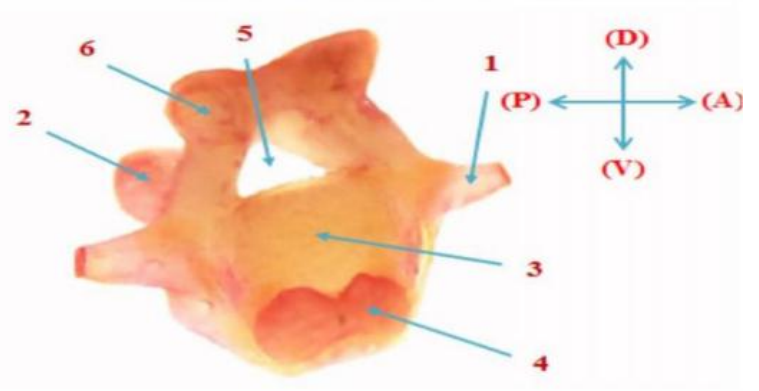

Figure 3. Mixture bone intact red-eared turtles (Trachemys scripta Elegans) females by staining Alizarin Red S-Alcian Blue (as seen from the anterior side or front view). Dorsal (D), Ventral (V), Anterior (A), posterior (P). Prosessus lateralis (1), Prezygapophysis (2), Centrum (3), Chevron (4), Canalis vertebra (5), Postzygapophysis (6).
In preparation red-eared turtles (Trachemys scripta elegans) females by staining Alizarin Red S-Alcian Blue (Figure 3) looks centrum and also canal vertebra in which there is the spinal cord, but the spinal cord is not seen because at the time of purification in $\mathrm{KOH}$ medulla spinal go missing with other organs than bone. Articulation between prezygapophysis and postzygapophysis serves to strengthen columna vertebral and excessive bending (Figure 3).

Between the vertebrae from one another are connected by cartilage tissue called the intervertebral disc. Intervertebral disc is attached to the surface of the vertebrae in the anterior and posterior, called the centrum. Prosessus lateral located on the right and left vertebral caudalis which serves as a regulator of the balance while in motion (Figure 4).

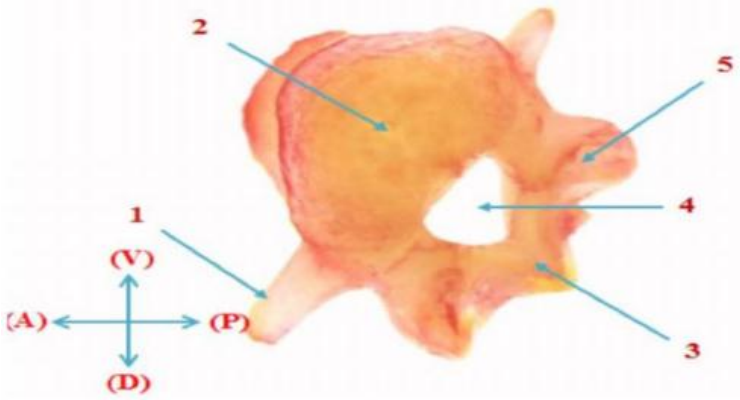

Figure 4. Mixture bone intact red-eared turtles (Trachemys scripta Elegans) females by staining Alizarin Red S-Alcian Blue (seen from the posterior or rear). Dorsal (D), Ventral (V), Anterior (A), posterior (P). Prosessus lateralis (1), Centrum (2), Arcus neural (3), Canalis vertebra (4), Prezygapophysis (5).

\section{Microscopic Observation Red-eared Turtle (Trachemys scripta elegans) Females}

In the longitudinal slices red-eared turtles (Trachemys scripta elegans) female with Hematoxylin eosin staining (HE) (Figure 5), the color purple is the core cell preparations stained by hematoxylin, while pink is a cytoplasmic eosin stained.

Muscles look pink because the color it with Hematoxylin eosin (HE). The skin on turtles is very thick. In red-eared turtles (Trachemys scripta elegans) female skin has scales thick tail. Muscles are divided or partitioned for their muscle segments (Figure 5).

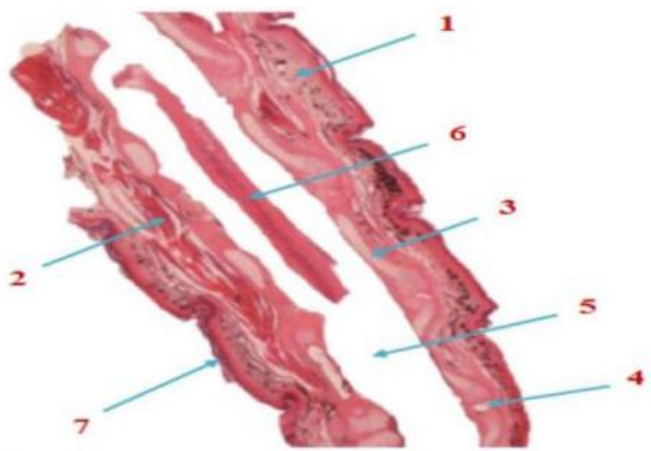

Figure 5. Sliced lengthwise turtle tail bone (Trachemys scripta elegans) female with Hematoxylin-eosin staining (HE) 4x10 magnification. Leather (1), Striated muscle (2), Bone (3), Intervertebral (4), Canalis vertebra (5), Spinal medulla (6), Scales (7). 
On the outer part of the tail vertebrae caudalis surrounded by muscle (Figure 6). Muscles that are contained in the Red-eared turtles (Trachemys scripta elegans) is a female-shaped striated muscle plain. This muscle to contract faster and stronger. In addition striated muscles are also very sensitive to stimulation. The muscles in the tail is composed of several myotube joined together to form myotomes. Between myotomes the one with the other myotomes myoseptum or separated by a septum. Meanwhile, between the muscle bundle with one another muscle file separated by a muscular septum. Observations on preparations slices of muscle transversely on Red-eared turtles (Trachemys scripta elegans) females by staining Hematoxylin-eosin (HE) (Figure 6) and Mallory acid fuchsin (Figure 11) shows that the muscle on Red-eared turtles (Trachemys scripta elegans) females segmented structure.

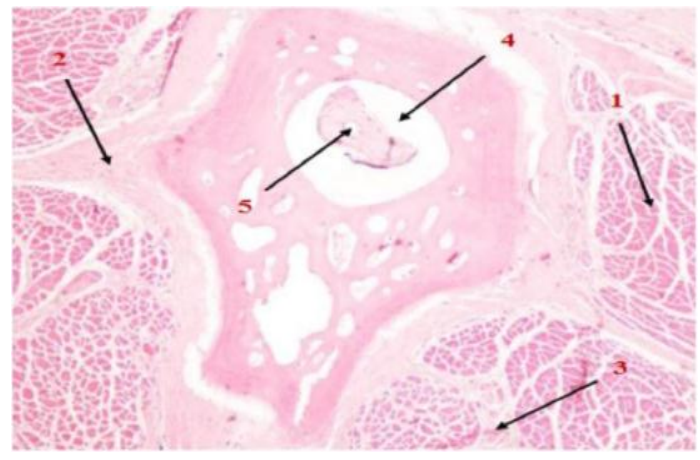

Figure 6. The cross sectional bone red-eared turtles (Trachemys scripta elegans) female with Hematoxylin-eosin staining (HE) 4x10 magnification. Striated muscle (1), Muscular septum (2), Myoseptum (3), Canalis vertebra (4), The spinal medulla (5).

In Red-eared turtles (Trachemys scripta elegans) females have thick scales attached to the skeletal muscle in the form of plain lying parallel to the vertebrae caudalis (Figure 7).

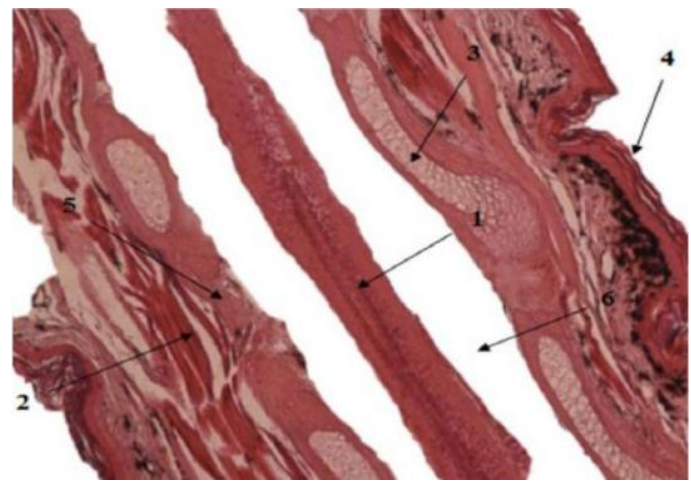

Figure 7. Sliced lengthwise red-eared turtle tail bone (Trachemys scripta elegans) female with Hematoxylin-eosin staining (HE) 10x10 magnification. Spinal cord (1), Striated muscle (2), Bone (3), Scales (4), Intervertebral (5), Canalis vertebra (6)

In the cross-sectional Red-eared turtles (Trachemys scripta elegans) have no female fat tissue perivertebral. Medulla spinalisnya immediately surrounded by the vertebral canal and muscle tissue (Figure 8). According to Syarifah (2012) on reptiles have the ability autotomy the outside of the vertebra surrounded by fatty tissue. The nucleus of cells of the bone Red-eared turtles (Trachemys scripta elegans) while the female purple pink plasma membrane (Figure 8).

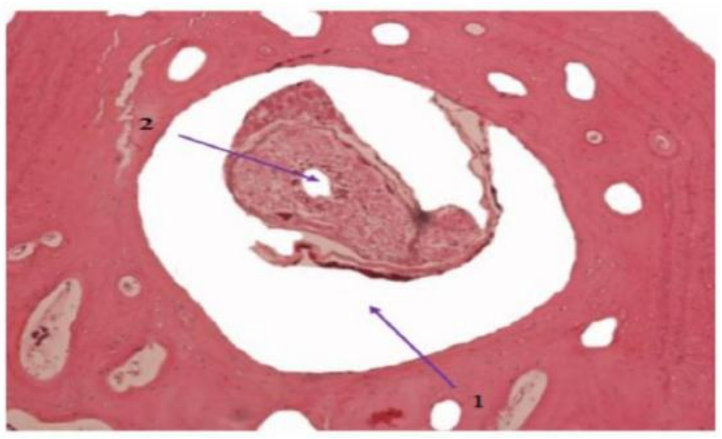

Figure 8. The cross sectional bone red-eared turtles (Trachemysscripta elegans) female with Hematoxylin eosin staining (HE) 10x10 magnification. Canalis vertebra (1), The spinal medulla (2).

Observations on preparations sliced lengthwise and crosswise bone in tail female red-eared turtle (Trachemys scripta elegans) with staining Hematoxylin-eosin (HE) (Figure 5) and Mallory acid fuchsin (Figure 9) shows that in the vertebra caudalis visible such as skin, striated muscle, bone, intervertebral, vertebral canal, and spinal cord.

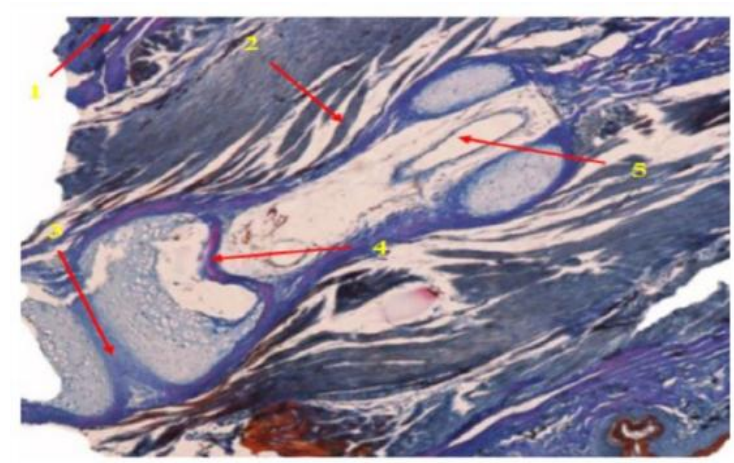

Figure 9. Sliced lengthwise red-eared turtle tail bone (Trachemys scripta elegans) female with acid fuchsin staining Mallory 4x10 magnification. Leather (1), Striated muscle (2), Intervertebral (3), Bones (4), Canalis vertebra (5).

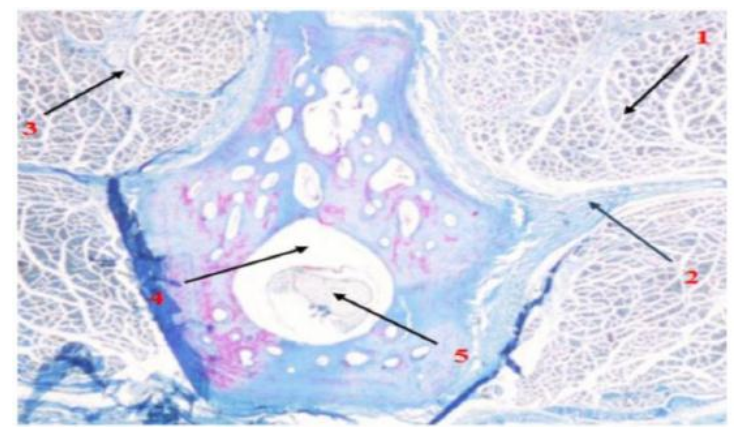

Figure 10. The cross sectional bone red-eared turtles (Trachemys scripto elegans) female with acid fuchsin staining Mallory 4x10 magnification. Striated muscle (1), Muscular septum (2), Myoseptum (3), Canalis vertebra (4), The spinal medulla (5). 
Muscles Red-eared ian turtles (Trachemys scripta elegans) is divided into 4 files female muscle file. Between the muscle file with a file other muscles are separated by a septum muscles. The spinal cord is located in the center surrounded by vertebral and vertebral canal (Figure 10).

In the longitudinal incision bone red-eared turtles (Trachemys scripta elegans) female with acid fuchsin Mallory staining showed collagen fibers bright blue, while the nucleus, cytoplasm, elastic fibers and fibrin in red. Erythrocytes and myelin fibers are colored yellow to orange. This shows the connective tissue that surrounds the red-eared turtles (Trachemys scripta elegans) females and all the connective tissue system serves as its function (Figure 11). In these pictures that myelin fibers orange, dark blue muscle while hyalin bluish material (Figure 11).

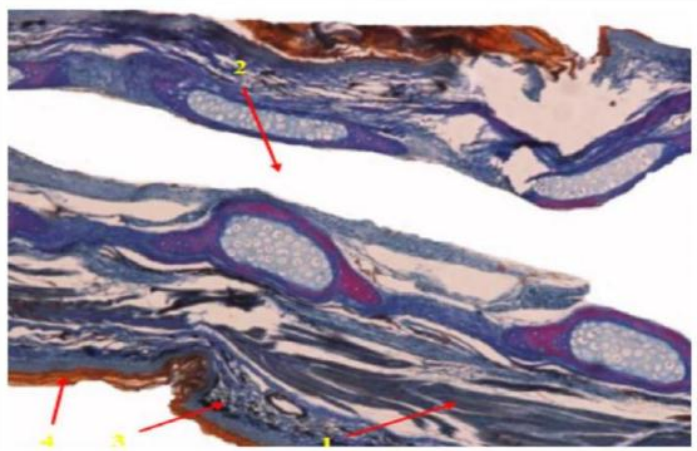

Figure 11. Sliced lengthwise turtle tail bone (Trachemys scripta elegans) female with acid fuchsin staining Mallory 10x10 magnification. Striated muscle (1), Canalis vertebra (2), Melanophore (3), Caucasian (4).

In the cross-sectional bone Red-eared turtles (Trachemys scripta elegans) females seen in blue, yellow to orange and red. This is because mallory acid fuchsin staining on a more specific staining for collagen fibers stained coloring of blue light, the core fibers and myelin stained yellow to orange (Figure 12).

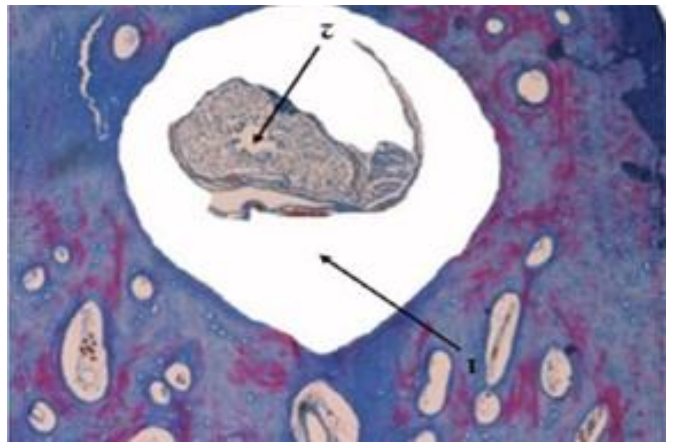

Figure 12. The cross sectional bone red-eared turtles (Trachemys scripta elegans) female with acid fuchsin staining Mallory 10x10 magnification. Canalis vertebra (1), The spinal medulla (2).

Some prosessus contained in the vertebrae, among others zygaphophysis consisting of prezygapophysis and postzygapophysis. Prezygapophysis an anterior bulge of the neural arch with articulation facing dorsomedial facies. While postzygapophysis a posterior protrusion of the neural arch with articulation facies facing ventrolateral (Winchester, 1965).

\section{CONCLUSIONS}

Based on observations and data analysis as well as the discussion that has been done, then obtained the following conclusions:

1. Vertebral caudalis Red-eared turtles (Trachemys scripta elegans) females composed of true bone, there is a lateral prosessus, prosessus dorsal, ventral prosessus, does not have the plain type amphicoelous autotomy and vertebrae.

2. Histology Red-eared turtles (Trachemys scripta elegans) females resemble other tail histological structure of the reptiles with some differences that only have 4 files do not have the muscle and fat tissue.

\section{REFERENCES}

Cann, J. 1978. Tortoises of Australia. Angus and Robertson. Sydney. p: 79.

Carr, AF, Jr. 1952. Handbook of Turtles. The Turtle of the United States, Canada and Baja California. Cornell University. Press, Ithaca, New York. p: 248-258.

Connor, MJ 1992. The Red-eared Slider. Trachemys scripta elegans. http://www.tortoise.org/archives/elegans.html. On October 3, 2012.

Das, I, R. Whitaker and Z. Whitaker, 1993. The World of Turtles and Crocodiles. National Book Trust, New Delhi. p: 64.

Encyclopedia Indonesia. Encyclopedia 1988. Indonesia Fauna Series. Reptiles and Amphibians. Editors Encyclopedia Indonesia. Mold I. p: 25-39.

Ernst, CH and RW Barbour, T. 1989. Turtles of the World. Smithsonian Institution Press. Washington DC and London.

Haecky, V. 1996. Water Turtle Care Sheet-Introduction, setups. http://www.turtlecare.net/setup.htm. On October 3, 2012.

Iverson, JB 1985. Checklist of the Turtles of the World with English Common Names. Herpetological Circular p: 1-14.

Iskandar, DT 2000. Turtles \& Crocodiles Indonesia and Papua New Guinea. PAL Media Citra. Bandung.

O'Keeffe, M. and Scott. 2006. Red-eared Slider Turtles. In Australia and New Zealand. Impact Status Management. BrisbaneQueensland.

Romer, AS and TS Parson. 1986. The Vertebrate Body. Sixth Edition. Saunders College Publishing. USA. p: 169-170.

Rakhmiati, 2012. The structure of Anatomy and Histology Tails and Tail Original Regenerat Cicak (Hemidactylus frenatus Schlegel, 1836). Essay. State Islamic University Sunan Kalijaga (Unpublished)

Suntoro, Handari. 1983. Staining Method. Work Bhratara Script. Jakarta. p: 221-233.

Syarifah, AZ 2012. Axial Skeleton calcification and Segmentation MuscularRegenerat Tailed Gecko (Gekko gecko Linnaeus, 1758). Essay. State Islamic University Sunan Kalijaga (Unpublished)

Wake, MH 1992. Hyman's Comparative Vertebrate Anatomy. Third Edition. The University of Chicago Press. Chicago. p: 194-197.

Weiss, L. and RO Greep. 1977. Histology. Fourth Edition. Mc Graw-Hill Book Company. USA. p: 206-239.

Young, JZ 1989. The Life of the vertebrates. Third Edition. Oxford University Press. Oxford.

Zug, GR 1993. Herpetology an Introductory Biology of Ampibians and Reptiles. Academic Press. Londo 